\title{
Psicooncología
}

ISSN: 1696-7240

\section{La experiencia de un grupo de apoyo en el proceso de duelo de familia- res de niños con cáncer. Un estudio cualitativo}

\author{
Anabel Melguizo-Garín ${ }^{1 *}$; Isabel Hombrados-Mendieta²; M José Martos-Méndez ${ }^{3}$
}

Recibido: 29 de noviembre de 2019 / Aceptado: 10 de enero de 2020

Resumen. Objetivo: El presente estudio se plantea analizar el discurso de un grupo de familiares en duelo por el fallecimiento de un menor con cáncer, que acudieron a los grupos de apoyo de Fundación Olivares en Málaga. Método: Análisis del discurso de los nueve familiares participantes en el grupo de apoyo. Resultados: Del discurso de dichos participantes se obtienen afirmaciones relacionadas con la mejora de la calidad de vida de estas personas a través del apoyo social, el bienestar emocional y la expresión emocional. Conclusión: El grupo de apoyo se perfila como una herramienta útil para familiares que están viviendo este proceso y facilita el apoyo social y la expresión y bienestar emocional. De este estudio preliminar se derivan importantes implicaciones prácticas.

Palabras clave: Padres de niños con cáncer; apoyo social; bienestar emocional; expresión emocional; duelo; grupos de apoyo.

\section{[en] The support group experience in the grieving process of family members of children with cancer. A qualitative study.}

\begin{abstract}
Objective: The present study proposes to analyze the discourse of a group of relatives in mourning for the death of a child with cancer, who attended the support groups of the Olivares Foundation in Malaga. Method: Analysis of the discourse of the nine family members participating in the support group. Results: From the discourse of these participants, statements related to the improvement of the quality of life of these people are obtained through social support, emotional wellbeing and emotional expression. Conclusion: The support group is emerging as a useful tool for family members who are living this process and facilitates social support and emotional expression and wellbeing. Important preliminary implications derive from this preliminary study.
\end{abstract}

Keywords: Parents of children with cancer; social support; emotional well-being; emotional expression; grief, support groups.

1 Anabel Melguizo-Garín. Departamento de Psicología Social. Facultad de Psicología. Universidad de Málaga. Campus Universitario de Teatinos s/n. 29071 Málaga (Spain)

E-mail: anamel@uma.es

2 Isabel Hombrados-Mendieta. Departamento de Psicología Social. Facultad de Psicología. Universidad de Málaga. Campus Universitario de Teatinos s/n. 29071 Málaga (Spain)

E-mail: mihombrados@uma.es

3 M José Martos-Méndez. Departamento de Psicología Social. Facultad de Psicología. Universidad de Málaga. Campus Universitario de Teatinos s/n. 29071 Málaga (Spain)

E-Mail:mjmartos@uma.es

* Dirección de correspondencia: Anabel Melguizo-Garín. Departamento de Psicología Social. Facultad de Psicología. Universidad de Malaga. Campus Universitario de Teatinos s/n. 29071 Malaga (Spain).

E-mail: anamel@uma.es 
Sumario: 1. Introducción 2. Objetivo 3. Método 4. Resultados 5. Conclusiones 6. Agradecimientos 7. Referencias bibliográficas.

Cómo citar: Melguizo-Garín A, Hombrados-Mendieta I, Martos-Méndez MJ. La experiencia de un grupo de apoyo en el proceso de duelo de familiares de niños con cáncer. Un estudio cualitativo. Psicooncología 2020;17:117-129. doi: 10.5209/psic.68245.

\section{Introducción}

El proceso de la enfermedad de un hijo con cáncer suele ser largo y estresante, son muchos los cuidadores que pasan años dedicados al cuidado del hijo con cáncer, dejando a un lado otros aspectos de sus vidas, no pudiendo prestar la atención que les gustaría en muchas ocasiones a la pareja, los otros hijos, el trabajo, las relaciones sociales e intereses personales. Un cáncer es un elemento desestabilizador para cualquier familia $^{(1)}$, pero es tras el doloroso acontecimiento de la muerte de un hijo con cáncer cuando se producen las mayores reacciones y sentimientos encontrados en el cuidador y los familiares. Es en este momento cuando se hacen necesarios los recursos que se puedan poner a disposición de estas familias, para que aquellas que así lo consideren puedan recibir atención especializada después del fallecimiento del hijo ${ }^{(2)}$. En este sentido, hay que decir que son muchas las entidades que ayudan a los niños y sus familias a lo largo de la enfermedad prestando apoyo psicosocial y económico, pero no son tantas las que ofrecen una estructura y espacio para las familias que han perdido a un hijo a causa del cáncer.

\section{El cáncer como enfermedad terminal}

La idea de pérdida y de duelo está presente desde el mismo momento del diagnóstico del cáncer y acompaña al paciente y a su familiar a lo largo de todo el proceso de enfermedad. Durante el diagnóstico surgen una serie de miedos asociados a la muerte, al dolor, a la incapacidad, a la alteración del esquema corporal, a ser abandonados, al cambio, y a la pérdida del proyecto de vida. La adaptación del paciente y de la familia a la enfermedad implica asumir las sucesivas pérdidas que se van presentando y en esencia el cáncer les hace a todos reflexionar sobre la vida, el presente, pasado y futuro $^{(3)}$. La muerte por cáncer suele ser un proceso largo, para el que no siempre se está preparado y algunas veces es vivido como el fracaso de una tecnología que por momentos hace olvidar que el ser humano después de todo es mortal. En ocasiones, sobreviene demasiado deprisa, de manera que no hay tiempo para adaptarse a la enfermedad y sus implicaciones, otras veces, la muerte ocurre después de muchos meses o años de sufrimiento y los familiares llegan agotados física y mentalmente, producto del cuidado que ha demandado el paciente ${ }^{(4)}$.

\section{Duelo y cáncer infantil}

La muerte de un ser querido es de los acontecimientos vitales más estresantes que puede afrontar el ser humano. El duelo se refiere al síndrome de sufrimiento emotivo que se observa en la mayoría de las personas que tienen alguna pérdida, y presenta etiología, sintomatología y curso similar a los del trastorno de adaptación ${ }^{(5)}$. En el 
caso de la pérdida afectiva por el fallecimiento de un ser querido, el duelo se refiere al conjunto de reacciones de tipo físico, emocional y social que se producen por el fallecimiento de una persona próxima y que pueden oscilar desde un sentimiento transitorio de tristeza hasta una desolación completa, que, en los casos más graves, puede durar años e incluso toda la vida ${ }^{(6)}$. Otra definición más extensa es la de Payás(7), según la cual el duelo es la respuesta natural a la pérdida de cualquier persona, cosa o valor con la que se ha construido un vínculo afectivo, y como tal, se trata de un proceso natural y humano y no de una enfermedad que haya que evitar o de la que haya que curarse. La expresión del duelo incluye reacciones, que muy a menudo se parecen a aquellas que acompañan a trastornos físicos, mentales o emocionales ${ }^{(8)}$.

Cada padre y cada madre de un niño diagnosticado con una enfermedad grave como es el cáncer reacciona de una manera determinada, en estas reacciones influyen muchos factores: tipo de cáncer, pronóstico y situación familiar entendiéndola en un amplio sentido, entre otros ${ }^{(9)}$. Una reacción que se repite en la mayoría de los padres es el fuerte impacto emocional tras conocer el diagnóstico, caracterizado por síntomas de ansiedad, nerviosismo, desasosiego, incertidumbre, estado de ánimo triste y desesperanza ${ }^{(10,11)}$. Desde la primera vez que escuchan la palabra cáncer, (palabra cargada de connotaciones negativas y relacionada con la muerte), la idea de la pérdida del hijo planea en la mente de los padres.

Este largo camino al que se enfrentan las familias con un hijo con cáncer no siempre acaba con la curación del niño, por el contrario, son muchas las ocasiones en las que tras años de cuidados y dedicación el hijo fallece. Ya durante la última fase de la enfermedad terminal la familia comienza a anticipar el duelo y las emociones y sentimientos relacionados con éste ${ }^{(12)}$. En este momento la intervención psicológica es entendida como apoyo a la familia para que expresen sus emociones, estén presentes en los cuidados y en los últimos momentos y no sientan que han perdido el control de la situación, así como resolver asuntos pendientes que queden con el hijo o relacionados con éste.

Tras la muerte de un hijo es cuando se producen las mayores reacciones y sentimientos encontrados en el cuidador y los familiares ${ }^{(13)}$; de alivio después de tantos años de lucha y sufrimiento, sobre todo si el proceso ha deteriorado mucho al hijo y se asume como irreversible, de culpa por no haber podido hacer nada más por el hijo, de soledad y desesperanza en el cuidador principal ya que después de tanto tiempo dedicado plenamente al hijo tras la muerte de éste siente que ya no existe nada más, de miedo ante la incertidumbre en el futuro, etc. ${ }^{(14)}$.

\section{Apoyo social y grupos de apoyo}

En estos momentos de la enfermedad la red de apoyo (familiares, amigos, vecinos, grupos de autoayuda) y el apoyo social que éstos prestan adquiere una gran importancia ${ }^{(15)}$. El apoyo social es un factor fundamental en la salud física y psíquica, de manera que las personas con buen apoyo social pueden tolerar mejor las situaciones de estrés ${ }^{(16,17)}$.

El apoyo social se define como los recursos sociales que las personas perciben que tienen disponibles ${ }^{(18)}$ y que les son proporcionados de manera formal por contextos o grupos profesionales o instituciones y asociaciones, o de manera informal por grupos de apoyo como familia, amigos y pareja. En resumen, el apoyo social se refiere a la 
percepción o la experiencia de que uno es amado y cuidado, estimado y valorado, y es parte de una red social de asistencia mutua ${ }^{(19,20)}$.

Los grupos de apoyo para familiares persiguen mejorar la calidad de vida de estas familias a través del apoyo social, la expresión emocional y el bienestar emocional. Todo ello se encuadra en un contexto en el que el familiar se siente escuchado y comprendido por otros familiares que están pasando por su misma situación, sin perjuicio de ser juzgados o no entendidos por los otros ${ }^{(7)}$, lo que facilita la percepción de apoyo y la expresión emocional. Y, por tanto, contribuye a mejorar la calidad de vida de estas personas.

Los beneficios de esta atención son muchos, las familias que han recibido apoyo durante el proceso de la enfermedad, tras el fatal desenlace no se sienten "solas y desamparadas"(21), se les sigue prestando apoyo una vez que el niño ya no está, este recurso es también un apoyo para las Unidades de Salud Mental de los diferentes centros públicos, ya que debido a las características propias de estas familias en duelo, requieren atención especializada en el ámbito de la Psicooncología, y por ello a veces se hace complicado ubicarlas en otras estructuras que ofrecen sus servicios de manera más generalizada.

\section{El análisis del discurso como herramienta de estudio psicosocial}

Analizar discursos es una tendencia que ha logrado importante aceptación en las Ciencias Humanas y Sociales ${ }^{(22)}$. El análisis del discurso (AD) se inscribe en lo que podríamos denominar el saber cualitativo, formando parte de lo que Valles ${ }^{(23)}$ llama el paradigma interpretativo. Lo característico del análisis del discurso y que le distingue de otras técnicas de investigación, es que se trata de una técnica que combina intrínsecamente, y de ahí su complejidad, la observación y producción de los datos, y la interpretación o análisis de los mismos ${ }^{(24)}$.

Los discursos, las verbalizaciones y los signos de diferente naturaleza que los acompañan (no sólo lingüísticos), tienen efectos sobre la realidad social ${ }^{(25)}$, es decir, el lenguaje no se considera solamente un vehículo para expresar y reflejar nuestras ideas, sino un factor que participa y tiene injerencia en la constitución de la realidad social $^{(26)}$.

Autores como Laclau y Mouffe ${ }^{(27)}$ postulan que lo discursivo es una dimensión crucial en el establecimiento de los vínculos y de las relaciones sociales. Y, por tanto, esta concepción activa del lenguaje también ha influido sobre la psicología, y en esa área, en los últimos veinte años el AD dejó de considerarse como una herramienta de importancia marginal para pasar a ser una perspectiva empleada frecuentemente ${ }^{(28)}$.

Este estudio se plantea como objetivo analizar los beneficios que tiene una experiencia de grupo de apoyo de familiares que han pasado por el fallecimiento de un niño con cáncer a través del análisis de los discursos que realizan dichos familiares durante las sesiones. En el duelo el contar con la posibilidad de acudir a un grupo de apoyo con personas que están en una situación parecida puede ayudar a manejar mejor el proceso. La aplicación del AD en esta realidad social es muy novedosa y aunque esta herramienta es ampliamente utilizada en las ciencias psicosociales aplicadas, en el contexto de nuestro estudio su aplicación es más escasa. Esta metodología cualitativa es en sí misma una fuente de conocimiento muy interesante 
cuando se trata de analizar y estudiar los discursos de los grupos y variables que se pueden relacionar con dichas verbalizaciones y puede ser complementada con posteriores estudios cuantitativos.

\section{Objetivo}

El objetivo principal que se persigue con este estudio es conocer si el grupo de apoyo es una herramienta útil para la mejora de la calidad de vida, la percepción de apoyo social, el bienestar emocional y la expresión emocional en estos familiares. Para ello, es necesario analizar los beneficios que tiene una experiencia de grupo de apoyo de familiares que han pasado por el fallecimiento de un niño con cáncer.

En este estudio en concreto vamos a utilizar la herramienta metodológica de análisis del discurso (AD), de naturaleza cualitativa, partiendo de la siguiente hipótesis:

A lo largo de las sesiones y al finalizar el grupo de apoyo, los discursos de los familiares relacionados con el apoyo social, la expresión emocional y el bienestar irán en aumento, lo cual puede ser una evidencia de la utilidad del grupo de apoyo.

\section{Método}

Definición de las variables del AD:

Un paso importante del método, a pesar de que no existe una única pauta o guía para ponerlo en práctica, es definir las variables que vamos a analizar en los discursos transcritos de los participantes durante las sesiones. El objetivo de esta etapa del análisis es tener claro qué es lo que estamos analizando y cuáles son las verbalizaciones que tendremos que cuantificar dentro del discurso.

La definición de las variables fue la siguiente:

Calidad de vida: entendida como satisfacción vital, se define como una valoración global que la persona hace sobre su vida, comparando lo que ha conseguido, sus logros, con lo que esperaba obtener, sus expectativas ${ }^{(29)}$.

Apoyo social: se refiere a la percepción o la experiencia de que uno es amado y cuidado, estimado y valorado, y es parte de una red social de asistencia mutua ${ }^{(19,20)}$.

Bienestar emocional: capacidad de sentir emociones evaluadas como positivas y reconfortantes, es una dimensión de la calidad de vida ${ }^{(30,31)}$.

Expresión emocional: capacidad para verbalizar y comunicar las propias emociones, validarlas y permitirlas. Este concepto ha venido de la mano del desarrollo del concepto de inteligencia emocional. Ésta se define como las destrezas con las que se consigue: a) percibir los estados emocionales, asumiéndolos como tales y expresándolos convenientemente; comprender correctamente la naturaleza de dichos estados emocionales, b) regularlos, impidiendo sus efectos negativos y aprovechando sus aspectos positivos para adaptarnos satisfactoriamente a cada situación y c) ser capaces de hacer lo mismo con los estados emocionales de las demás personas ${ }^{(32)}$. 


\section{Participantes}

El grupo de apoyo ha estado constituido por nueve familiares de niños fallecidos por cáncer (dos padres, una hermana mayor de edad y seis madres) que acuden a Fundación Olivares de Málaga, la edad media de los participantes es de 40 años, y el tiempo medio desde el fallecimiento del menor es de un año. La dinámica grupal se llevó a cabo en catorce sesiones semanales de dos horas de duración. Las variables analizadas fueron la calidad de vida, la percepción de apoyo social, el bienestar emocional y la expresión emocional.

\section{Procedimiento}

Cuando hablamos de grupo de apoyo no hay una forma única válida de funcionamiento en el grupo ${ }^{(7)}$. Las sesiones a pesar de ser semidirigidas y dejar un margen de actuación espontánea de los participantes bastante amplio, seguían una estructura más o menos fija que fue la siguiente:

- Apertura: Ronda de expresión libre.

- Pausa.

- Actividad dirigida por la facilitadora del grupo con la finalidad de promover la ventilación emocional, validar el proceso de cada uno y cohesionar el grupo a través de la experiencia compartida. Algunas de las actividades propuestas fueron las siguientes: 1 . Actividades artísticas que apoyen al proceso de expresión emocional $^{(33)}$, algunas de estas técnicas son propias de la arteterapia, entendiendo por arteterapia el proceso de simbolización acompañada ${ }^{(34)}$. El objetivo de estas técnicas es favorecer y asistir los procesos creativos de los participantes, ajustándolos a sus ritmos, necesidades y capacidades, de forma que sean capaces de ir proyectando su mundo interior y sus procesos emocionales en la creación plástica $^{(35)}$. 2. El uso de técnicas de cohesión grupales para mejorar la relación del grupo ${ }^{(12)}$. Se realizaron dinámicas de grupo cuyo objetivo es el fomento de los lazos entre los participantes y así fortalecer el apoyo que presta el grupo. Las dinámicas de grupo tienen que ver con los procesos que se producen en los grupos de diferente naturaleza y con las técnicas que se utilizan para intervenir en los mismos ${ }^{(36)}$. Las técnicas de dinámica de grupo son también definidas como aquellas situaciones colectivas que son guiadas por un coordinador que plantea simulaciones a problemas o conflictos que hace que el grupo se movilice para conocerse mejor de manera grupal y de manera individual, de este modo, nacen nuevas formas de comportamiento ${ }^{(37)}$. 3. También se utilizó la psicoeducación para entender mejor el proceso de duelo ${ }^{(38)}$, esta herramienta aplicada al proceso de duelo ayuda a proporcionar información a los participantes acerca de los mecanismos que se producen en el duelo, se comentan reacciones emocionales, fases por la que se transita, en definitiva, en qué consiste el proceso por el que están pasando y porqué se produce, y esto permite a los padres reinterpretar su propio proceso teniendo en cuenta la nueva información ${ }^{(2)}$. 4. Otra cuestión importante es hacer referencia en estos grupos a las diferencias en los modelos de afrontamiento del proceso de duelo ${ }^{(39)}$, existen diferentes estrategias de afrontamiento que los padres que están pasando por esta situación pueden poner en práctica, abordarlas en el grupo y ponerlas de relieve contribuye a un mejor manejo del proceso ${ }^{(7,12)}$. Hablamos de habilidades de afrontamiento 
o tipos de afrontamiento para referirnos a acciones específicas que la persona pone en marcha para enfrentarse a una situación concreta ${ }^{(40)}$, en la literatura se han recogido diferentes tipos de afrontamiento. Podemos hablar de modelo de afrontamiento centrado en la emoción y modelo de afrontamiento centrado en el problema, en el primero se intenta regular la emoción que provoca distrés, y el segundo se centra en manejar el problema que origina esa emoción. Otros modelos hablan de estrategias de afrontamiento aproximativas (se confronta el problema) y estrategias de afrontamiento evitativas (se escapa del problema) ${ }^{(41)}$. Es importante resaltar que no existe unas estrategias de afrontamiento mejor que otras y que dependerá de las consecuencias, si la persona obtiene beneficios con dicha estrategia, su uso es apropiado en ese momento ${ }^{(40)}$. Explicar estos modelos con un objetivo psicoeducativo puede ayudar a validar el proceso de duelo de los participantes del grupo.

- Cierre de la sesión: Ronda de preguntas dirigidas por el terapeuta para conocer cómo se sienten tras la sesión, verbalización de las emociones sentidas después del trabajo en grupo.

Las sesiones siguieron el guion temático que se detalla a continuación:

- $1^{\mathrm{o}}$ sesión: bienvenida a los participantes que han vivido una pérdida reciente. Iniciación, presentación y ronda grupal.

- $2^{\circ}$ sesión: fases del duelo, descripción y compartir grupal. Cada uno encuentra la fase del duelo en la que se halla. Explicar y educar sobre las diferentes etapas del duelo, acompañar a transitar por todas esas etapas, compartir sus propias etapas de afrontamiento.

- $3^{\circ}$ sesión: dar salida a lo reprimido mediante lo artístico. A través de técnicas artísticas, dar lugar a que se exprese lo reprimido. Provocar la exteriorización de emociones a través de técnicas artísticas, buscar el afrontamiento de la situación.

- $4^{\circ}$ sesión: trabajos grupales de autoapoyo. Técnicas de fusión del grupo para generar mayor confianza.

- $5^{\circ}, 6^{\circ}, 7^{\circ}, 8^{\circ}, 9^{\circ}$ y $10^{\circ}$ sesión: afrontamiento de las fases del duelo. Cada participante comparte su forma de afrontar. Técnicas y educación del afrontamiento del duelo.

- $11^{\circ}, 12^{\circ}, 13^{\circ}$ y $14^{\circ}$ : cierre de las sesiones. Sucesivas sesiones para seguir trabajando en estas líneas. Sentirse apoyados en este proceso y apoyar a otros participantes del grupo en este proceso.

Una vez que finalizaron las sesiones se dio por concluido el grupo de apoyo y no se descarta poner en marcha un nuevo grupo de apoyo mutuo y que el grupo objeto de este estudio pase a ser un grupo de autoayuda.

Se llevaron a cabo transcripciones de las verbalizaciones emitidas por los participantes al cierre de cada sesión y, además, se apoyó esta transcripción con un cuestionario breve a modo de autoinforme con una pregunta abierta que los participantes compilaban al final de las sesiones (véase Anexo 1).

\section{Análisis de las verbalizaciones}

Finalizadas las sesiones se realizó un análisis del discurso de los nueve participantes del grupo de apoyo al duelo, a través de la transcripción de las sesiones y la 
cuantificación de las afirmaciones emitidas por cada uno de ellos relacionadas con: calidad de vida, percepción de apoyo social, bienestar y expresión emocional, estas variables en el proceso se convirtieron en categorías en las que se fue clasificando la información. Para explicar el análisis realizado, se han incluido algunos fragmentos representativos de las variables. Se ha garantizado la cantidad (saturación) y la calidad de la información, recogiendo datos en todas las sesiones realizadas y de todos los participantes. Conociendo las tres formas básicas de codificación en el análisis de contenido cualitativo (inductiva, deductiva y mixta) ${ }^{(42)}$ hemos elegido la segunda, ya que buscamos en el discurso aquellas verbalizaciones que se relacionan con las categorías (variables) planteadas a priori.

A continuación, se transcriben algunas de las verbalizaciones relacionadas con las variables que hemos definido en este estudio.

\section{Resultados}

Análisis del discurso de las verbalizaciones de los participantes:

\section{- $1^{\text {a }}$ variable CALIDAD DE VIDA}

"Me siento mucho mejor", "Me encuentro mejor desde que vengo al grupo", "En el grupo me siento bien", "Cuando vengo al grupo me siento tranquila y en paz".

\section{- $2^{\text {a }}$ variable APOYO SOCIAL}

"En el grupo me siento comprendido," "Me está ayudando mucho asistir al grupo", "Creo que vosotros entendéis como me siento", "Me siento apoyado por el grupo", "Nadie me va a devolver a mi hijo, pero me reconforta poder contar con vosotros".

\section{- $3^{\text {a }}$ variable BIENESTAR EMOCIONAL}

"El rato que vengo aquí es un alivio y salgo mucho mejor", "Compartir con vosotros me ayuda a estar más positivo en mi vida", "Me siento bien cuando alguno de nosotros se siente mejor escuchándonos y compartiendo nuestra experiencia", "Desde que vengo estoy más tranquila con mi familia y me siento menos culpable cuando me río".

\section{- $4^{\text {a }}$ variable EXPRESIÓN EMOCIONAL}

"En el grupo consigo hablar de lo que me pasa con libertad", "No me siento juzgado", "Sé que lo que yo siento también lo pueden sentir los otros miembros del grupo y eso me anima a seguir".

Estas verbalizaciones se relacionan con las diferentes variables y a lo largo de las sesiones van aumentando en todos los participantes y todos consideran que es muy útil y positiva la experiencia del grupo para trabajar el duelo. 


\section{Conclusiones}

El análisis del discurso de las verbalizaciones de los participantes puede ser una herramienta para conocer si la experiencia de grupo de apoyo para estos familiares se perfila como herramienta útil para ellos ${ }^{(7)}$. Podemos concluir que el trabajo en un grupo de apoyo con familiares que han pasado por el fallecimiento de un niño con cáncer constituye una herramienta facilitadora de apoyo, permite desarrollar un mejor manejo de las emociones, mejora la percepción de apoyo social y la expresión emocional, aumenta las verbalizaciones de tipo positivo relacionadas con el apoyo social y el bienestar a lo largo de las sesiones y crea un sentimiento colectivo de utilidad del grupo y de sentirse parte de una colectividad mayor ${ }^{(43,44)}$.

Estos resultados pueden relacionarse con que los logros propiciados por los grupos de apoyo ayudan a que el proceso del duelo se desarrolle de la manera más normalizada posible, alejando a la persona en duelo de aislarse socialmente o de no expresar sus emociones ${ }^{(45)}$. Además, estos grupos intentan devolverles a los participantes el control sobre sus propias vidas, y los sujetos se convierten en participantes activos en el desarrollo de recursos, en el afrontamiento de situaciones problemáticas y en la provisión de apoyo ${ }^{(44)}$.

A través del apoyo mutuo y expresión emocional la persona no sólo es ayudada por el grupo, sino que puede ayudar convirtiendo su experiencia personal en un potencial de ayuda para sí misma y para los otros participantes del grupo ${ }^{(46)}$.

El estudio es preliminar ya que con éste se pretende conocer si el grupo de apoyo con estas características se perfila como una herramienta útil para las familias en esta situación, por ello, es necesario tomar los resultados con cautela, debido a que la metodología es cualitativa y no es posible generalizar los resultados. Además, la muestra es limitada y para futuras experiencias sería conveniente elaborar un método de análisis cuantitativo a través del análisis de escalas sobre las variables a estudio.

El apoyo social tiene un efecto amortiguador del estrés e influye de manera positiva sobre la salud ${ }^{(47)}$, sería muy interesante profundizar en los efectos que el apoyo social y estos grupos de apoyo mutuo tienen sobre el estrés y la salud de los familiares participantes y si, además, teniendo en cuenta el efecto amortiguador, son una fuente de prevención de posibles complicaciones en el proceso del duelo ${ }^{(48)}$. Teniendo en cuenta los resultados de este estudio inicial, sería positivo continuar en esta línea de trabajo para futuras investigaciones.

Pese a las limitaciones, este estudio nos alienta a continuar en esta línea de trabajo con las familias y profundizar más en los beneficios y, para ello, nos planteamos la posibilidad de llevar a cabo estudios en los que se profundice en más variables relacionadas con la utilidad de los grupos de apoyo en el proceso de duelo.

\section{Agradecimientos:}

A la Fundación Olivares y los padres y familiares participantes en el grupo de apoyo. 


\section{Anexo 1.}

\section{Grupo de Apoyo Mutuo}

Fecha de la sesión:

Cuestionario breve de evaluación de la sesión.

Tras la ronda de cierre en la que hemos compartido nuestras impresiones sobre cómo ha ido la sesión y en qué pensáis que os está ayudando y siendo útil, por favor, en el espacio que tienes aquí, describe brevemente esas impresiones que has compartido con el grupo, así como cualquier otra cuestión que quieras señalar sobre la sesión. 


\section{Referencias bibliográficas:}

1. Die-Trill M. Efectos psicosociales del cáncer en el enfermo pediátrico y su familia. Primeras jornadas internacionales de atención multidisciplinar al niño con cáncer; Valencia: ASPANION, 1993.p.97-114

2. Payás A. Las tareas del duelo. Psicoterapia de duelo desde un modelo integrativorelacional. Barcelona: Paidós Ibérica, 2010.

3. Barreto P, Yi P, Soler, C. Predictores de duelo complicado. Psicooncología 2008;5:383400.

4. Barreto P, Yi P, Soler C, Martínez E, Fombuena M, Aldana C, Espinar V. Factores protectores del duelo complicado. Comunicación presentada en el VI Congreso de la Asociación Latinoamericana de Cuidados Paliativos. Lima. 2008.

5. Elkin G. Psiquiatría clínica. México: Masson, 2000.

6. Echeburúa E. Superar un trauma. El tratamiento de las víctimas de sucesos violentos. Madrid: Pirámide, 2004.

7. Payás A. Intervención grupal en duelo. En Camps C, Sánchez P T, editors. Duelo en oncología. Madrid: Sociedad Española de Oncología Médica, 2007. p. 169-82.

8. Worden W. Tratamiento del duelo: asesoramiento psicológico y terapia. Ed. Paidós, 2008.

9. García-Fernández E, González García-Portilla M P, Saiz-Martínez P, Llorca-Ramón J, Bobes-García J. Impacto psicopatológico (ansioso-depresivo) en padres de niños con tumores hematológicos. Anales de Psiquiatría 1999; 15: 153-8.

10. Grau C. La integración escolar del niño con neoplasias. Barcelona: CEAC, 1993.

11. Grau C, Fernández M. Familia y enfermedad pediátrica. An Sist San Navarra 2010; 33: 2003-12. [Acceso 1 de febrero de 2019]. Disponible en: http://scielo.isciii.es/scielo. php?script=sci_arttext\&pid=S113766272010000300008\&lng=es.

12. Bermejo J C, Sánchez E J. Grupos de ayuda mutua en el duelo: eficacia y perspectivas. Mapfre Medicina 2007; 18: 82-90.

13. Neimeyer R A. Aprender de la pérdida. Barcelona: Paidós, 2002.

14. Neimeyer A. Lessons of loss. Psychoeducational resources. Florida: Keystone Heights, 2000.

15. Melguizo-Garín A, Martos-Méndez MJ, Hombrados-Mendieta, I. Influencia del apoyo social sobre el estrés y la satisfacción vital en padres de niños con cáncer desde una perspectiva multidimensional. Psicooncología 2019; 16:25-42. doi: 10.5209/PSIC.63646.

16. Choi E K, Yoon S J, Kim J H, Park H J, Kim J Y, Yu E S. Depression and distress in caregivers of children with brain tumors undergoing treatment: psychosocial factors as moderators. Psychooncology 2016; 25: 544-50. doi: 10.1002/pon.3962

17. Hoekstra-Weebers J E, Jaspers J P, Kamps W A, Klip E C. Psychological adaptation and social support of parents of pediatric cancer patients: A prospective longitudinal study. J Pediatr Psychol 2001; 26: 225-35. doi: 10.1093/jpepsy/26.4.225

18. Gottlieb B H, Bergen A E. Social support concepts and measures. J Psychosom Res 2010; 69: 511-20. doi: 10.1016/j.jpsychores.2009.10.001

19. Taylor S E, Sherman D K, Kim H S, Jarcho J, Takagi K, Dunagan M S. Culture and social support: Who seeks it and why? J Pers Soc Psychol 2004; 87: 354-62. doi: 10.1037/00223514.87.3.354

20. Wills T A, Shinar O. Measuring perceived and received social support. En Cohen S, Underwood L G, Gottlieb B H, editors. Social support measurement and intervention: A guide for health and social scientists. New York: Oxford University Press, 2000, p.86135. doi: 10.1093/med:psych/9780195126709.003.0004 
21. Pulido E, Martinez C, Morán M, Ortiz A, Campillos M, Gutiérrez D, Elvira J. Guía de intervención Psicosocial y cuidados de duelo. Cordoba: Guías de intervención psicológica en servicios sociales comunitarios, 2009. p. 4-91.

22. Santander P. Por qué y cómo hacer Análisis de Discurso. Cinta moebio 2011; 41: $207-$ 224. Disponible en: www.moebio.uchile.cl/41/santander.html

23. Valles M. Técnicas cualitativas de investigación social. Madrid: Síntesis, 2000.

24. Andréu J. Las técnicas de análisis de contenido: una revisión actualizada. Sevilla: Fundación Centro de Estudios Andaluces: 2002.

25. Verón E. Semiosis social. Buenos Aires: Gedisa, 1998.

26. Austin J. Cómo hacer cosas con palabras. Barcelona: Paidós, 1982.

27. Laclau E, Mouffe Ch. Hegemonía y estrategia socialista. México: FCE, 2004.

28. Antaki C, Billig M, Potter J. El Análisis del discurso implica analizar: Crítica de seis atajos analíticos. Athenea Digital. Revista de Pensamiento e Investigación Social 2003: 1:14-35. [Acceso 20 de diciembre de 2019] Disponible en: http://psicologiasocial.uab.es/athenea/ index.php/atheneaDigital/index

29. Pavot W, Diener E D, Colvin C R, Sandvik E. Further validation of the Satisfaction with Life Scale: Evidence for the cross-method convergence of well-being measures. J Pers Assess 1991; 57: 149-61.

30. Arostegui I. Calidad de vida y retraso mental. Bilbao: Mensajero, 2002.

31. Schalock R L, Verdugo M A. Calidad de vida. Manual para profesionales de la educación, salud y servicios sociales. Madrid: Alianza, 2003.

32. Cerezo M V, Ortiz-Tallo M, Cardenal V. Expresión de emociones y bienestar en un grupo de mujeres con cáncer de mama: una intervención psicológica. Rev Latinoam Psicol 2009; 41:131-40.

33. Collette N. Arteterapia y cáncer. Psicooncología 2011; 8: 81-99. doi: 10.5209/rev_ PSIC.2011.v8.n1.7

34. Klein J.P. La creación como proceso de transformación. Arteterapia: Papeles de arteterapia y educación artística para la inclusión social. 2006; 1: 11-8.

35. Gutierrez-Ajamil E. Arteterapia familiar en oncología pediátrica. Psicooncología 2018; 15:133-51. doi: 10.5209/PSIC.59183.

36. Canto JM, Montilla V. Dinámica de grupos y autoconciencia emocional. Perspectivas teóricas y ejercicios prácticos. Archidona (Málaga): Ediciones Aljibe, 2008.

37. Antons K. Práctica de la dinámica de grupos. Barcelona: Hérder, 1990.

38. Sanz J M, García I M, Carbajo E. Tratamiento del duelo en Salud Mental: Una experiencia grupal. Revista de Psicoterapia 2014: 25; 115-33.

39. Bermejo J C, Magaña M, Villacieros M, Carabias R, Serrano I. Estrategias de afrontamiento y resiliencia como factores mediadores de duelo complicado. Revista de Psicoterapia 2012: 22; 85-95.

40. Lazarus R, Folkman, S. Stress, appraisal and coping. New York: Springer, 1984.

41. Rodriguez-Marín J. Psicología Social de la Salud. Madrid: Síntesis Psicología, 1996.

42. Moreno-Jiménez MP, Ríos-Rodríguez ML. "Sin nosotras el mundo no se mueve". Mujeres inmigrantes en el contexto laboral español. Athenea Digital 2012: 12(2); 3-31. [Acceso 20 de diciembre de 2019] Disponible en: http://psicologiasocial.uab.es/athenea/ index.php/atheneaDigital/article/view/Moreno-Rios

43. Hombrados-Mendieta I, Martimportugués, C. Los grupos de apoyo social y autoayuda. En Hombrados-Mendieta, I, García MA, López T, editors. Intervención Social y Comunitaria. Ediciones Aljibe, 2006; 139-149. 
44. Hombrados-Mendieta I, García M, Martimportugués C. Grupos de apoyo social con personas mayores: una propuesta metodológica de desarrollo y evaluación. Anuario de Psicología 2004: 35; 347-370.

45. Ospina-Velasco A M. Los grupos de apoyo: una alternativa para la elaboración del duelo. Prospectiva. Revista de Trabajo Social e Intervención Social 2003; 8: 171-84.

46. Riessman, F. Redefining self-help, police and practice. San Francisco: Jossey-Bass Publishers, 1995.

47. Martos-Méndez MJ. Enfermedades crónicas y adherencia terapéutica. Relevancia del apoyo social. Editorial Universidad de Almería, 2010.

48. Prigerson HG, Horowitz MJ, Jacobs SC, Parkes CM, Aslan M, Goodkin K, et al., Prolonged Grief Disorder: Psychometric Validation of Criteria Proposed for DSM-V and ICD-11. PLOS Med 2009; 6:8. doi: 10.1371/journal.pmed.1000121 
